temperatures naturaliy calls to mind Prof. Giglioli's most remarkable experiments with regard to the actions of poisons, both gaseous and liquid, on seeds. An account of them was given in NATURE, I882, p. 328 , and 1895, p. 544 . He found that dried seeds of Medicago sativa, although exposed to the prolonged action of gases such as oxygen, chlorine, nitric oxide, \&c., and of poisonous fluids, e.g. alcohol, corrosive alcohol, \&c., retained their power of germination. In some of his experiments the time of immersion of the seeds in the poison was so prolonged (many years) that the supposition of the non-penetration appeared precluded. I have repeated Giglioli's experiments with several species, and found, as he did, that some seeds can withstand the action of poisons while others cannot. Seeds of Medicago sativa were exposed from I0-30 days to the action of methylated spirit, spirit saturated with mercuric chloride and with picric acid without their powers of germination being noticeably affected. Similarly, seeds of Papaver Rhoeas, $P$. somniferum and Schizopetalon Walkeri resisted the action of spirit, but were apparently killed by corrosive alcohol. Papaver R'hoeas germinated after two days' immersion in chloroform and two days in spirit. On the other hand, seeds of Nicotiana Tabacum, Linaria reticulata, Gypsophila paniculata and Calandrina umbellatum did not germinate after immersion in spirit.

The following experiment shows, I think, that this astonish ing resistance to poisons is not due to the quiescent state or stability of the protoplasm of the seed, but to the imperviousnes of the seed-coat. A large number of seeds of Medicago sativa were taken, and half of them were punctured with the prick of a needle. All were then desiccated, and after desiccation immersed some in spirit, some in spirit and mercuric chloride and some in spirit and picric acid. It was then found that the intact seeds germinated in large quantities even after immersion in the poisonous fluids, while the punctured seeds germinated in no case after immersion. In a control experiment it was found that the punctured seeds both before and after desiccation germinated freely. It would appear, then, that when the penetration of the poison was secured the effect was to destroy the vitality of the seed.

Botanical Laboratory, Trinity College, Dublin.

An Instance of Adaptation among the Deer

My friend Major C. S. Cumberland has just brought from Manipur the head and hind-foot of a deer the latter of which affords an interesting instance of adaptation to environment. The deer in question is the Manipuri representative of the thamin (Cervnes eldi) of Burma, an animal inhabiting open tree jungle. The Manipur valley is, however, a huge swamp, and the thamin of that locality have developed a peculiar modification in the foot which enables them to walk with ease in such ground. In the Burmese thamin the under surface of the hind pasterns is covered with hair in the ordinary manner, and the animal walks entirely on the main hoofs, keeping the pasterns much elevated. In the Manipuri thamin on the other hand, the under surface of the pasterns is covered with a hard, horny, bare skin, which immediately above each hoof has almost the consistency of horn, and is practically continuous with the hoof itself. Moreover, so far as can be determined from comparison with a mounted specimen of the Burmese form, the pasterns are very considerably longer than in the latter In walking, according to the account given by Major Cumberland, the foot is much bent, so that the animal walks on nearly the whole of the under surface of the pasterns, and thus gains a firn support on the yielding morass.

Assuming this feature to be constant (as Major Cumberland believes to be the case), the Manipuri thamin appears entitled to rank as a distinct local form, for which the name $C$. eidi cornipes will be appropriate, Major Cumberland's specimens standing as the type.

But, quite apart from this minor point, the specimens are of especial interest as showing a previously unknown mode by which ruminants may adapt themselves to a life in swamps. In the well-known instance of the sitatunga antelope of Africa a sufficiently large surface of support is afforded by a lengthening of the hoofs ; in the present case the hoofs remain of the norma length, and support is obtained by the animal walking on the under surface of the pasterns, which is specially hardened. It is, in fact, an incipient instance of the reversion of a digitigrade animal to the plantigrade progression of its swamp-dwelling ancestors.

R. LYDEKKER.

\section{Snow Conditions in the Antarctic}

THE meteorologist of my expedition has unfortunately given a somewhat incorrect idea about the snow conditions at Cape Adare. He reports, namely, that there is a very small snow fall at the sea-level. This is, in my opinion, not the case. But his mistake is excusable and easily explicable ; of course, being a young Tasmanian and not previously having seen ice and snow, a devotee to his instrument, took down in his note book the evidence of a usual snow gauge. Snow seldom or never fell in the Antarctic except during heavy gales, and it must be clear to anybody familiar with snow that a snow gauge of the ordinary type is worse than useless during heavy gales. Although Cape Adare itself and the peninsula on which we lived were almost free of snow in the open, we had more than ten feet of snow to the leeward of our hut at Camp Ridley, and undoubtedly there would have been still more had the huts been higher. This indicates, of course, that much snow fell, but it was blown away as well from the promontory at Cape Adare as from the unfortunate snow gauge. In my opinion a very heavy snow fall takes place within the Antarctic circle. And I believe that the strong gales within the Antarctic circle generally are local and that these snow bared dark promontories are the very homes of the Antarctic gales, while those places where no dark land is to be seen probably are unmolested by great atmospheric disturbances and are therefore covered in heavy snow. From time to time in the pack ice I have passed through distances where the ice was covered in several yards of loose snow. This I noticed as well on my first voyage in 1894 as during my last expedition. I will therefore use the opportunity to warn the coming expeditions from not providing against the difficulties which a very heavy snow fall incurs for sledge parties within the Antarctic circle. C. E. BORCHGREVINK. (Commander British Antarctic Expedition, 1898-1900)

Douglas Lodge, Bromley, Kent, July 6.

\section{PHOTOGRAPHIC AND PHOTOMETRIC SURVEYS OF THE STARS. ${ }^{1}$}

EVERYONE will naturally wish to offer words of hearty congratulation to Sir David Gill and his able coadjutor, Prof. Kapteyn, on the completion of the Cape Durchmusterung, of which the third and last volume has recently appeared. Some twenty years since, when the capacity of celestial pholography was practically an unknown factor, Sir David Gill proposed to himself to complete a survey of the southern hemisphere by means of photographic star maps. The original conception was a tolerably modest one. Sir David Gill's idea was simply to prepare from these maps a working catalogue of stars to facilitate the meridian zone observations, after the programme of the Astronomische Gesellschaft, but "to avoid the repetition of such an arduous undertaking as Argelander's Durchmusterung as a preliminary step." How the original plan was extended and grew, till the results fill three bulky volumes, exceeding Argelander's work both in number of stars and in accuracy of observation, he has himself told in the introduction to the first part, to which we have already referred (NATURE, vol. lvii. p. 513). Very rapidly has the work gone on once all preliminary difficulties were removed, and now the astronomers of the Cape and of Groningen see their work completed on a uniform plan within a moderate space of time, with an accuracy which approaches that attaching to the older so-called "Precision Catalogues," together with the means existing for the determination in special instances of star places with even greater accuracy. For though we have spoken of the completion of the work,

1 "The Cape Photographic Durchmusterung for the Equinox 1875." By David Gill, C.B., LL.D., F.R.S., \&c., His Majesty's Astronomer at the Cape, and J. C. Kapteyn, Sc.D., \&c., Professor of Astronomy at Groningen. Part iii. Zones - 53 to $-89^{\circ}$. Pp. $88+67$ r. (Edinburgh : Neill and Co., 1900.)

" A Photometric Durchmusterung, including all Stars of the Magnitude 7.5 and brighter North of Declination -40 , obtained with the Meridia: of the Harvard College Observatory. Pp. 330. (Cambridge, U.S.A., Igor.)

$$
\text { NO. I654, VOL. 64] }
$$


this is to be understood in a limited sense. The discussion of the catalogue is about to begin. Such discussion will include the examination and detection of errors in the "Precision Catalogues," the search for, and discovery of, stars with large and unsuspected proper motions, and the formation of a catalogue of variable stars for the southern hemisphere. Further, the course of the work has disclosed the existence of a possible systensatic difference of colour in stars, depending on the galactic latitude, and intimately connected with this inquiry is the investigation of the systematic corrections which should be applied to the magnitudes derived from the Cape plates to ensure one uniform system, photographically considered, or to connect the photographic and optical magnitudes. A revision conducted on such ample lines is a task of only less magnitude than that of the construction of the catalogue itself, while the importance and interest are even greater. That the same competent hands will carry such a discussion to a final issue will be the hope and the expectation of all astronomers.

The introduction supplied by Prof. Kapteyn to the third volume cannot possess the novelty and the interest which naturally attaches to that accompanying the first volume of the annals. In that it was necessary to detail his scheme of measuring the plates and effecting the reduction; he also sketched the results at which he had arrived by comparison with the work made on the meridian by other astronomers, pursuing similar but less extensive methods. The subsequent volumes have had to record the mechanical accumulation of the places and magnitudes of stars observed by the same method, till we have piled up for us the enormous total of 454,384 stars, catalogued within an area of I3,9 I square degrees, embraced in the district between the South Pole and the parallel of $19^{\circ}$ south declination. The greater richness of the southern skies is shown by the fact that this number is only slightly less than that contained in the joint Durchmusterung of Argelander and Schonfeld, extending from the North Pole to $23^{\circ}$ south declination. Further, this richness has increased as the observations have been carried polewards, the maximum being reached in the zone $48^{\circ}-58^{\circ} \mathrm{S}$. decl. In the first section, comprising the zone $18^{\circ}-37^{\circ} \mathrm{S}$. decl., the average number of stars to a square degree was $25^{\circ} 43$ (NATURE, loc. cit.) ; for the whole the average number is $32^{\circ} 66$, or more than double that of the Northern Bonn Durchmusterung. But in a discussion involving relative density of aggregation the vexed question of the maintenance of a uniform standard of magnitude throughout the whole survey enters with perplexing uncertainty. On this point Prof. Kapteyn entertains views into which we do not care to enter too minutely, for we are yet awaiting lis complete answer to sundry criticisms which have been advanced. We are certainly inclined to follow him in his assertion that if two or three tenths of a magnitude be deducted from the estimates we shall obtain the limit of photographic magnitude to which the stars of the catalogue are certainly practically complete, and that consequently we may assume the whole catalogue to embrace all stars down to 9.2 mag.

There is, however, the other and more thorny question, which touches on the relative chemical activity of stars in different parts of the sky, which it is not so easy to answer. Prof. Kapteyn puts the question thus. To what limit of magnitude would the plates be found complete were the magnitudes of the Cape Durchmusterung reduced to a homogeneous set of photographic magnitudes for the whole sky? The answer which he offers is that the Durchmusterung will be found practically complete in or near the Milky Way, to stars which in the scales of Schonfeld, of Gould and of Thome are of the magnitude $9 \cdot 5$, and for the rest of the sky to stars actinically equivalent to these. The vagueness of this reply is due to the peculiar feature that Prof. Kapteyn's discussions have disclosed, and whose complete explanation is not yet forthcoming. The measurement of a great number of plates has satisfactorily shown that the law expressing the growth of star density depending on proximity to the Milky Way differs essentially from that exhibited in the optical observations of Schonfeld and Gould. For this fact two explanations, equally plausible, can be offered. Prof. Kapteyn's contention is that there is a real difference in the colours of the stars as the Milky Way is approached, and that the increase of blueness leads to increasing discrepancies in the differences between photographic and visual magnitude, amounting roughly to $0^{\circ}$ or mag. for each degree of galactic latitude. On the other hand, the ground for the observed inequality may be due to systematic errors in assigning the optical magnitudes to stars under the different condition in the method of selection of the stars, when they pass slowly in the sparser regions of the sky, and when the richer regions are being observed. The tendency might very well be, from the greater time at the disposal of the observer in the first case, to observe fainter stars than when he finds his field crowded with passing objects. Of course, both views were fully admitted by Prof. Kapteyn, but he considered he had sufficient evidence to establish his case, and though he acknowledges the force of the arguments which have been brought against him, he is still inclined to maintain his view. The fuller discussion is one of those points which have been left for future investigation so as not to delay the completion of the main work, but if the Groningen astronomer can obtain support for his theory it may have an important bearing on our views concerning the cosmical arrangement of the stars.

The accuracy of the stars' coordinates remains practically the same as in the earlier volumes, and from a comparison with Gould's meridian places may be given as follows :-

$\begin{array}{ccc}\text { Declination. } & \begin{array}{c}\text { Prob. Error in } \\ \text { Right Ascension. }\end{array} & \begin{array}{l}\text { Prob. Error in } \\ \text { Declination. }\end{array} \\ -38^{\circ} \text { to }-58^{\circ} & \pm 0^{\circ} \cdot 288 & \pm 0^{\prime} \cdot 0444 \\ -58^{\circ} \text { to }-86^{\circ} & \pm\left(0^{\circ} \cdot 157+0^{s} \cdot 0764 \mathrm{sec} \delta\right) & \pm 0^{\prime} \cdot 0559\end{array}$

But the method of measurement of stars on the polar plate affords a better means of determining the degree of accuracy attainable on these plates, and of the possible service they are likely to render in settling questions of identity or of proper motion. On this plate the rectangular coordinates have been measured with the Repsold apparatus acquired by the astronomical laboratory at Groningen, and the measures reduced by comparison with all the stars (save one) common to the plate and to Gould. Making due allowance for proper motion and error of observation in the Cordoba places, the probable errors of the Cape positions are found to be in R.A. $\pm 0^{\prime \prime} \cdot 53$ (arc of great circle); in decl. $\pm 0^{\prime \prime} \cdot 76$.

Considering the shortness of the focal length ( 54 inches) such a result is extremely gratifying, and it seems likely, as anticipated by the authors, that many questions connected with the proper motion of the southern stars can be at once set at rest by an appeal to the original plates. Some such work seems to have been already begun, judging by the tables added to the volume, in which are shown instances of stars missing on the plates and yet given elsewhere, and of stars found on the plates not recorded in known catalogues. Such work requires infinite patience and care, and we can only once more congratulate the joint authors on the success that has attended their unwearied efforts to secure uniformity and accuracy.

The second work under notice also exhibits the results of continued labour pursued with persistence and success. Photometry has so long been a feature in the researches at Harvard College Observatory, and so many successive volumes have detailed the method of observing, that on

$$
\text { NO. I 654, VOL. 64] }
$$


the present occasion the Director contents himself with a very short preface, but which gives evidence of the same untiring energy which marked the earlier volumes. For example, we are told that between 1891 and 1898 no less than 473,216 photometric settings were made with the meridian photometer, nearly all by the Director himself. The object of this heavy undertaking was to determine the magnitude of all stars brighter than 7.5 situated north of $-40^{\circ}$ declination. In the early days of magnitude work the Director did not propose to pass the limit of $-30^{\circ}$. This restriction was perhaps necessary on account of the smaller photometer employed, but to overstep it may also indicate that the Director feels himself now competent to cope with the difficult questions arising from the extinction of light in our atmosphere. For, although the Durchmusterung does not aim at completeness beyond $-40^{\circ}$, a good many stars, reaching to even within one degree of the Harvard horizon, have been included. Such measures are necessarily frequently discordant among themselves and do not agree with the estimates made in the southern hemisphere, but the discussion of all the discordant residuals, from whatever source arising, is deferred till the appearance of another volume. A difference of $0.65 \mathrm{mag}$. from the mean has been selected as marking the limit of discordant measures.

It will be noticed that this photometric survey covers no inconsiderable portion of the area that has been examined by Kapteyn. The whole of the first volume of the Cape Durchmusterung, $-18^{\circ}$ to $-37^{\circ}$, is included, and should therefore furnish at once enlarged material for the examination of the systematic differences between photographic and visual magnitudes. Further, the meridian Pickering photometer is at present at the Arequipa Observatory, having been dismounted in September 1898 , and the energetic Prof. Bailey is presumably using the same instrument at the southern station. Care has been taken to interchange the observers at Harvard so as to supply the means of reducing the observations on a uniform system, and thus continuing the Harvard survey to the Southern Pole. We may therefore look forward to the rapid acquisition of further data which will not only afford better values for the constants of reduction of the Cape plates, but exhibit in an unmistakable manner, though it may not solve, the perplexing difficulties to which we have alluded. Certainly, if energetic prosecution of the observations is of avail, the matter could not be in better hands than those of the Directors of Harvard and the Cape Observatories.

W. E. P.

THE TREATMENT OF DISEASE BY LIGHT.

PHOTOTHERAPY, or the treatment of disease by light, has now, thanks to Prof. Finsen of Copenhagen, a recognised place in the domain of therapeutics. Finsen's first paper on the subject was published in 1893 . In it he showed that the chemical or ultra-violet rays of the spectrum have a definite effect upon the course of small-pox, and he proposed that patients suffering from this disease should be kept in rooms from which the chemical rays of light were excluded by means of red curtains or red glass, in the same way that a photographer excludes these rays from his plates and paper. In an ordinary case of smallpox treated under the usual conditions, the eruption passes from the vesicular to the suppurative or pus-forming stage, and this condition is most marked upon the face and hands, the parts most exposed to light. It is in consequence of the destruction of the skin attendant upon the suppuration that the face and hands are so commonly the seat of hideous scars. Finsen's suggestion has been carried out with considerable success. In nearly every case in which the patient was kept in red light from the onset of the disease, there has been found to be a marked

No. I654, vOL. 64] change in the course of the eruption. The suppuration and its attendant secondary fever have been almost, if not entirely, abolished, and as a result the patients recover with little, if any, scarring.

Finsen's next researches were made upon the action of light as an irritant, and they are of extreme interest to the biologist. It will suffice here to say that he found that the animal organism, especially in creatures which prefer to dwell in the dark, is markedly irritated by the chemical rays, while the other parts of the spectrum are non-irritant. From this he was led to investigate the effects of light upon bacteria. Here the field had already been occupied by Downes and Blunt, who, in I 878 , in a paper read before the Royal Society, showed that the chemical rays are bactericidal. Duclaux, Arloing and others have worked upon the same lines and confirmed their results. It therefore seemed probable that superficial diseases of the skin caused by bacteria could be cured by the application of light. Of these, one of the most important and most intractable is lupus. Finsen, however, argued that the intensity of ordinary sunlight is obviously insufficient to kill the microbes as they lie in the skin, for lupus is particularly a disease of the face, which is more exposed to the sun than any other part. He therefore tried the effect of concentrating the light by means of lenses, cutting out the red and ultra-red rays by a blue medium. He found that cultures of microorganisms in vitro were much more powerfully influenced by the concentrated rays. The sun's rays concentrated by the apparatus to be presently described were fifteen times stronger than ordinary sunlight. Powerful electric arc lights were also tried, and with a lamp of from 35 to 50 amperres the effect was similar to that of the sun, or even greater.

The next point to be determined was the penetrative power of light. For this purpose small sealed tubes containing silver salts were placed under the skin of animals and exposed to the concentrated light, and the silver was found to be blackened.

The effect of the blood circulating in the tissues was next demonstrated by a very ingenious experiment. A piece of photographic paper was placed behind the ear, and the outside of the lobule was exposed to the light. In about five minutes the paper was blackened. The experiment was then tried with the ear compressed between two pieces of glass so that it was rendered bloodless. The photographic paper was blackened by the light in twenty seconds. The absence of the red colouring matter of the blood allowed the chemical rays to penetrate with great ease.

The apparatus devised by Finsen for the treatment of lupus by the sun's rays (Fig. I) consists of a large hollow planoconvex lens, filled with an ammoniacal solution of sulphate of copper and mounted upon a fork-like metal stand, so arranged that the lens can be moved about a horizontal and also round a vertical axis, and lowerer and raised at will. The filtered sun's rays are focussed upon the area of skin to be treated, and at this spot is placed the compression apparatus. This is a very flat cylinder made of two plates of rock crystal fixed in a metal ring. Through the compression apparatus passes a current of cold water, so that the instrument is used to render the part to be treated bloodless and also to cool it. The pressure apparatus is held on the skin by a nurse throughout the whole sitting, which lasts one hour or a little more. The spot treated at each sitting is about the size of a sixpence.

The electric light apparatus (Fig. 2) is much larger and more complicated. Attached to a strong metal ring round a large arc lamp, of 30,000 to 35,000 candle-power, are four long cylinders like telescopes. Each telescope consists of two parts. The upper part, closed at each end by rock crystal lenses, makes the divergent rays of the arc light parallel, and the lower piece brings the rays thus 